\title{
Chemotherapy enhances programmed cell death 1/ligand 1 expression via TGF- $\beta$ induced epithelial mesenchymal transition in non-small cell lung cancer
}

\author{
SOICHIRO FUNAKI, YASUSHI SHINTANI, TOMOHIRO KAWAMURA, \\ RYU KANZAKI, MASATO MINAMI and MEINOSHIN OKUMURA \\ Department of General Thoracic Surgery, Osaka University Graduate School of Medicine, Osaka 565-0897, Japan
}

Received April 2, 2017; Accepted July 25, 2017

DOI: $10.3892 /$ or.2017.5894

\begin{abstract}
In cancer immunology, the programmed cell death 1-programmed cell death 1/ligand 1 (PD-1/PD-L1) pathway plays a major role. Anti-PD-1 and anti-PD-L1 antibodies provide reliable immunotherapy when given as treatment for various types of malignancy including lung cancer. PD-L1 expression in cancer cells has been reported to be a predictive factor for the therapeutic effects of immunotherapy. However, the mechanism of PD-L1 expression remains unclear. Another key process in cancer progression is epithelial-mesenchymal transition (EMT). In the present study, we investigated the mechanism of PD-L1 expression as well as changes in its expression during the EMT process in non-small cell lung cancer (NSCLC). In this study, A549 cells underwent EMT by treatment with TGF- $\beta$ or chemotherapeutic agents and then PD-L1 expression was evaluated. The alterations of PD-L1 expression was also examined during the reverse EMT process; mesenchymal-epithelial transition (MET). The relationship between for PD-L1 expression and EMT status in clinical specimens with NSCLC after induction chemotherapy were analyzed by immunohistochemical staining. We found that PD-L1 expression was upregulated following TGF- $\beta$ induction; in contrast, it was downregulated by TGF- $\beta$ receptor-kinase inhibitors and the MET process. Furthermore, chemo-treatment increased TGF- $\beta$ expression and enhances PD-L1 expression via autocrine TGF- $\beta$ induced EMT. Analysis of clinical samples revealed a significant relationship between PD-L1 expression and EMT status $(\mathrm{P}<0.05)$. In conclusion, our results suggest that PD-L1 expression is regulated by TGF- $\beta$ induced EMT and enhanced by chemo-treatment via the chemo-induced TGF- $\beta$ signaling. The anti-PD-1/PD-L1
\end{abstract}

Correspondence to: Dr Soichiro Funaki, Department of General Thoracic Surgery, Osaka University Graduate School of Medicine, L-5 2-2 Yamadaoka Suita-city, Osaka 565-0897, Japan

E-mail: funaki@thoracic.med.osaka-u.ac.jp

Key words: non-small cell lung cancer, PD-1/PD-L1, epithelialmesenchymal transition, induction chemotherapy, TGF- $\beta$ blockade may provide more effective anticancer activities in combination with chemotherapy in NSCLC.

\section{Introduction}

Non-small cell lung cancer (NSCLC) is a leading cause of cancer-related death in most of the industrial countries. Chemotherapy, radiation therapy and surgery are the main therapeutic options. Despite these therapies, the prognosis for NSCLC has not improved as expected, and the mortality rate remains high. Therefore, additional therapeutic options are needed.

Recently, cancer immunotherapy has been developed and shown promising results against some malignancies in clinical trials (1-4). One of immuno-check-point proteins, the programmed cell death 1/programmed cell death 1/ligand 1 (PD-1/PD-L1) pathway has attract attention as playing a main role in cancer immunology (5). PD-1 (also known as CD279) is a receptor expressed on immune cells, and when combined with its ligand, PD-L1 (also known as CD274) expressed on cancer cells, induces the immunosuppression of cancer cells and blocks the attack by host immunity (5). Anti-PD-1/PD-L1 immunotherapies have recently been developed and has shown promising results against several malignancies (1-4). The expression of PD-L1 in cancer cells reportedly is a useful predictive factor for the therapeutic effect of anti-PD-1 or antiPD-L1 antibody immunotherapies (6).

However, the mechanism of PD-L1 expression in cancer cells remains unclear. In addition, immunotherapy has other problems to be solved (e.g. the selection of suitable patients and the timing of administration of the immunotherapy). Elucidation of the mechanism of PD-L1 expression may provide useful information to solve those problems.

Epithelial-mesenchymal transition (EMT) is a key process in cancer progression and is induced by several factors, including transforming growth factor (TGF- $\beta$ ). We previously showed that chemo-treatment increased TGF- $\beta$ expression in human adenocarcinoma cell lines, and this autocrine TGF- $\beta$ lead to higher malignant characteristics of cancer cells via EMT process $(7,8)$. We also revealed the clinical significance of the EMT in NSCLC after induction chemotherapy $(7,8)$. In the present study, we explored the mechanism by which 
TGF- $\beta$ controls PD-L1 expression and elucidated the clinical relationships between the PD-L1 expression and EMT status in NSCLC after induction chemotherapy.

\section{Materials and methods}

Cell culture, reagents and antibodies. The human lung adenocarcinoma cell lines A549 and NCI-H358 were purchased from the American Type Culture Collection (ATCC; Manassas, VA, USA) and maintained in RPMI-1640 medium with $10 \%$ fetal bovine serum (FBS) and streptomycin and penicillin. Carboplatin was purchased from Sigma-Aldrich (St. Louis, MO, USA; cat, no. C2538) and TGF- $\beta 1$ was from R\&D Systems (Minneapolis, MN, USA; cat. no. 240-B). SB 431542, a specific and selective inhibitor of TGF- $\beta 1$ receptor kinase inhibitor, was purchased from Tocris Bioscience (St. Louis, MO, USA; cat. no. 1614).

Reversion assay [mesenchymal-epithelial transition assay (MET)]. To determine whether there is a causal relationship between PD-L1 expression EMT, reversion assay was performed. We analyzed the gene expression of PD-L1 and $\mathrm{N}$-cadherin in A549 cells under MET (EMT reverse process). First, we induced EMT in the cells by using culture medium including TGF- $\beta 1(1 \mathrm{ng} / \mathrm{ml})$ for 3 days, and then we promoted MET by changing to culture medium without TGF- $\beta 1$.

Immunohistochemical staining analysis of EMT status and PD-L1 expression in clinical samples. IHC staining was performed as follows. Formalin-fixed paraffin-embedded tissue sections of non-small cell lung cancer from patients who underwent surgical resection after induction chemotherapy were deparaffinized and rehydrated. For antigen retrieval, the sections were brought to boil in $1 \mathrm{mM}$ EDTA pH 8.0 and then maintained for $15 \mathrm{~min}$ at a sub-boiling temperature. EMT status was evaluated according to N-cadherin, E cadherin and TGF- $\beta 1$ staining intensities. The evaluation of EMT status and PD-L1 were based on a previous study (9). In brief, the stained specimens were scored in a semi-quantitative manner (H score): the staining percentages $(0-100 \%)$ and the intensity 0 (no staining), +1 (weak staining), +2 (distinct staining), +3 (very strong staining) $\mathrm{H}$ score was calculated by multiplying the percentage by the intensity. In addition, $\mathrm{H}$ score was classified as 0 (score $<10),+1(\geq 10$ or $<30)$, or $+2(\geq 30$ or $<70),+3$ $(>70)$. We defined a positive change in EMT status as either or both $\mathrm{H}$ score classifications decrease in staining for E-cadherin and increase in the staining for $\mathrm{N}$-cadherin, respectively, between the biopsy samples before induction chemotherapy and the surgical samples after induction chemotherapy (CT). The PD-L1 immunohistochemistry was evaluated based on the method described by Koh et al (9). Briefly, the intensity and proportion of membranous and/or cytoplasmic staining in tumor cells were scored as follows: 0 , negative; 1 , weak or moderate in $<10 \%$ of tumor cells; 2 , moderate in $\geq 10 \%$ of tumor cells; 3 , strong (more intense than alveolar macrophages for PD-L1) in $\geq 10 \%$ of tumor cells. Cases with scores of 2 or 3 were deemed positive for PD-L1 expression. The antibody used for immunohistochemistry (IHC) were as follows: monoclonal mouse anti-human N-cadherin (6G11/M3613,1/50; Dako, Glostrup, Denmark), monoclonal mouse anti-human
Table I. Patients characteristics.

\begin{tabular}{lr}
\hline Variables & $\mathrm{N}$ \\
\hline Age (mean years) & 63.1 \\
Sex & \\
Male & 22 \\
Female & 6 \\
Clinical stage & \\
II & 3 \\
III & 25 \\
Pathological stage & \\
I & 9 \\
II & 6 \\
III & 13 \\
Histopathology & \\
Adenocarcinoma & 14 \\
Squamous cell carcinoma & 12 \\
Others & 2 \\
Chemotherapy & \\
1st & \\
CDDP & \\
CBDCA & 13 \\
2nd & 15 \\
ETP & \\
PTX & \\
TXT & \\
VDS & \\
VNR & \\
Surgical procedure & 23 \\
Pobectomy & 2 \\
Bi-lobectomy & \\
\hline
\end{tabular}

CDDP, cisplatin; CBDCA, carboplatin; ETP, etoposide; PTX, paclitaxel; TXT, docetaxel; VDS, vindesine; VNR, vinorelbine.

E-cadherin (NCH-38/M3612; Dako), polyclonal rabbit antihuman TGF- $\beta 1$ (ab66043; Abcam, Cambridge, UK) and polyclonal rabbit anti-human PD-L1 (EIL3N/13684, 1/200; Cell Signaling Technology, Inc., Danvers, MA, USA).

Reverse transcription polymerase chain reaction (RT-PCR). Total RNA was isolated from cell lines by using an RNeasy Mini kit (Qiagen, Tokyo, Japan). Real-time RT-PCR was conducted with a TaqMan assay; the relative expression levels were calculated by the comparative $C_{t}$ method. The TaqMan gene assays (Applied Biosystems, Carlsbad, CA, USA) for GAPDH (Hs02758991_g1), E-cadherin (Hs01023894_m1), N-cadherin (Hs00983056_m1), PD-L1 (Hs01125301_m1) and TGF- $\beta$ (Hs00998133_m1) were used. All experiments were performed in triplicate and the results are presented as means $\pm \mathrm{SD}$. The significance of differences between the untreated cells and the treated cells was tested with the Mann-Whitney U test. 

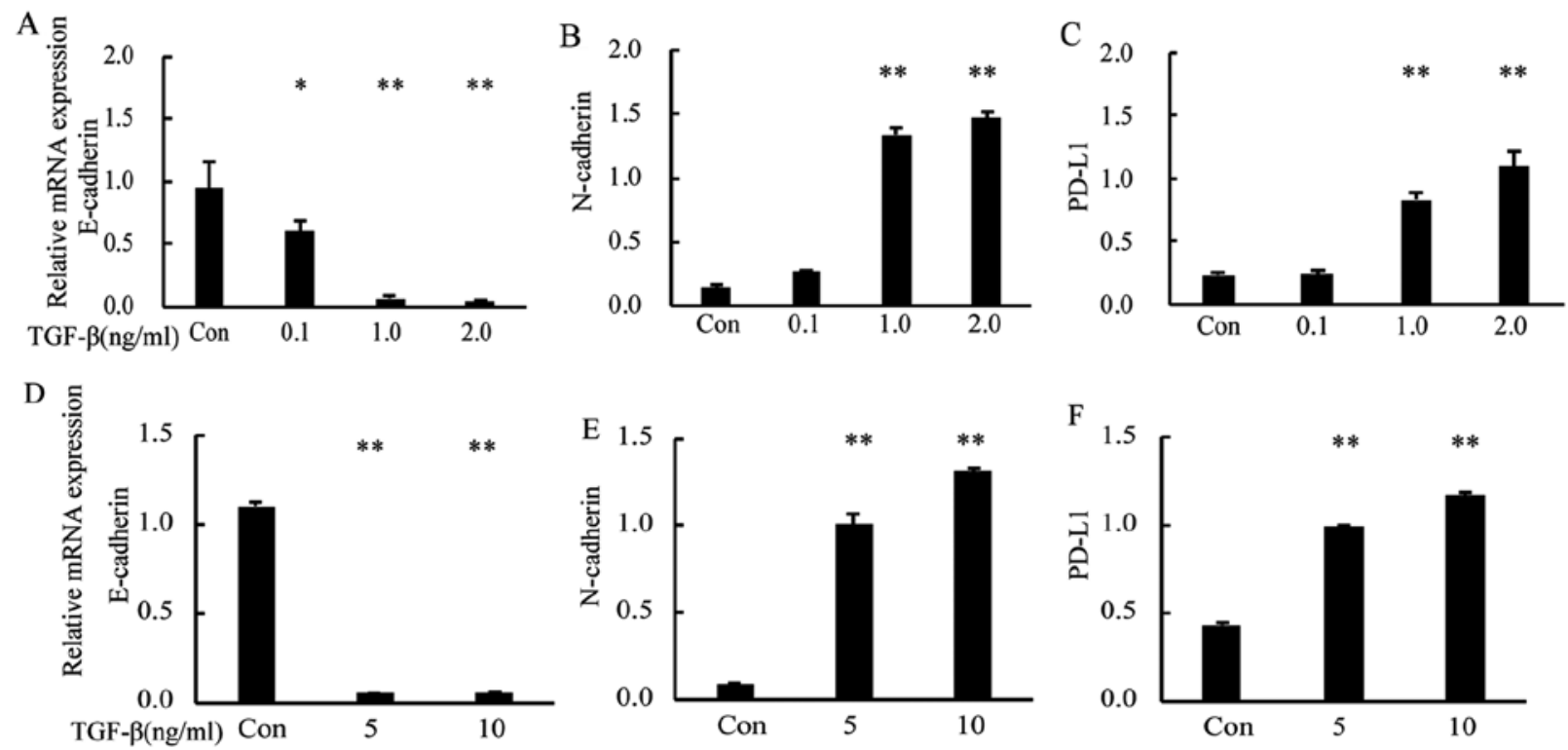

Figure 1. Effect of TGF- $\beta 1$ treatment on gene expression. (A-C) RT-PCR analysis of expression of the genes encoding E-cadherin (E-cad), N-cadherin (N-cad) and PD-L1 in A549 human lung adenocarcinoma cells following RPMI alone (Con; control) and TGF- $\beta 1$ treatment; mRNA levels were normalized to the levels of GAPDH mRNA; 0.1, 1.0 and 2.0 indicate treatment with TGF- $\beta 1$ at $0.1,1.0$ and $2.0 \mathrm{ng} / \mathrm{ml}$, respectively. ("P $<0.05$ vs. Con and ${ }^{* *} \mathrm{P}<0.01$ vs. Con). (D-F) Human Caucasian bronchioalveolar carcinoma H 358 (NCI-H358) cells were not treated (Con) or treated with TGF- $\beta 1$ (5-10 ng/ml) for 2 days. Then, total RNA was extracted and real-time RT-PCR was performed to detect E-cadherin, N-cadherin and PD-L1 mRNA. ( ${ }^{* *} \mathrm{P}<0.01$ vs. Con).

Western blot analysis. Monolayers of cultured cells were treated with TGF- $\beta$ or carboplatin, and the proteins were extracted with RIPA buffer (Cell Signaling Technology; cat. no. 9806). Cell extracts were separated with sodium dodecyl sulfate-polyacrylamide gel electrophoresis (SDS-PAGE) and immunoblotted as previously described $(10,11)$. Western blotting after extraction of membrane proteins was performed by using a Cell Surface Protein Isolation kit (P74008; Takara Bio, Tokyo, Japan). The following antibodies were used for detection: mouse anti-human E-cadherin (monoclonal, M106, $2 \mu \mathrm{g} / \mathrm{ml}$; Takara), mouse anti-human N-cadherin (monoclonal, sc-59987; Santa Cruz Biotechnology, Santa Cruz, CA, USA), rabbit anti-human GAPDH (monoclonal, G9545; SigmaAldrich) and rabbit anti-human TGF- $\beta$ (polyclonal ab66043; Abcam).

Study population. Twenty-eight patients underwent induction chemotherapy and pulmonary resection between 1996 and 2010 at the Osaka University Hospital. Osaka University Hospital Review Board approved this retrospective study, and written informed consent for this retrospective study and surgery was obtained from each patient. Each patient received two cycles of cisplatin- or carboplatin-based chemotherapy every 4 weeks in one of three regimens: cisplatin at $80 \mathrm{mg} / \mathrm{m}^{2}$ on day 1 and vindesine at $3 \mathrm{mg} / \mathrm{m}^{2}$ on days 1 and 8 , with or without mitomycin at $8 \mathrm{mg} / \mathrm{m}^{2}$ on day $1(\mathrm{PV}(\mathrm{M})$ regimen), cisplatin at $80 \mathrm{mg} / \mathrm{m}^{2}$ on day 1 and vinorelbine at $20 \mathrm{mg} / \mathrm{m}^{2}$ on days 1 and 8 (nPV regimen), or cisplatin at $80 \mathrm{mg} / \mathrm{m}^{2}$ on day 1 and docetaxel at $60 \mathrm{mg} / \mathrm{m}^{2}$ on day 1 (DP regimen) (Table I). A surgical resection was performed 6-8 weeks after induction chemotherapy.

Statistical design and data analysis. A $\chi^{2}$ test, MannWhitney $\mathrm{U}$ test, or repeated-measures analysis of variance

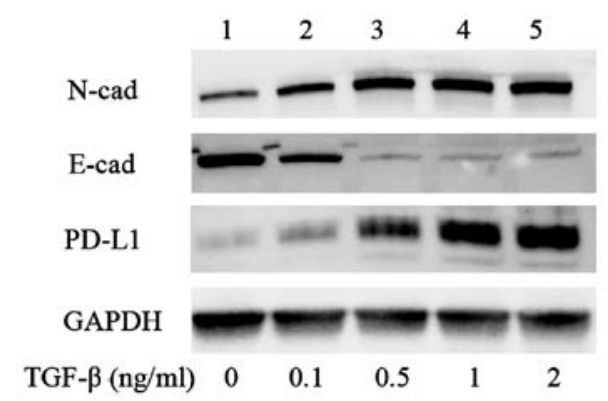

Figure 2. Western blot analysis PD-L1 and EMT marker protein after TGF- $\beta 1$ treatment. Western blot analysis of the indicated proteins following TGF- $\beta 1$ treatment; $0.1,0.5,1.0$ and 2.0 indicate treatment with TGF- $\beta 1$ at $0.1,0.5,1.0$ and $2.0 \mathrm{ng} / \mathrm{ml}$, respectively.

were used to compare the RT-PCR results. The correlations between PD-L1 IHC status and EMT markers were analyzed by the Pearson's Chi-squared test. Disease-free survival (DFS) and overall survival (OS) were analyzed by using the KaplanMeier method, and the log-rank test was used to compare the survival distributions of subgroups. All statistical analyses were performed by using the JMP version 11 for Windows (SAS Institute, Inc., Cary, NC, USA). $\mathrm{P}<0.05$ is considered to be statistically significant.

\section{Results}

$P D-L 1$ is enhanced in TGF- $\beta$ induced EMT and decreased in MET induced by removal of TGF- $\beta$ stimulation. To examine the relationship between PD-L1 and EMT status, we induced EMT in A549 and NCI-H358 cells by using TGF- $\beta 1$ at $0.1-2 \mathrm{ng} / \mathrm{m}$. RT-PCR analysis showed that, after TGF- $\beta 1$ treatment, the gene expression of E-cadherin (as the epithelial marker) was downregulated compared with that in control 

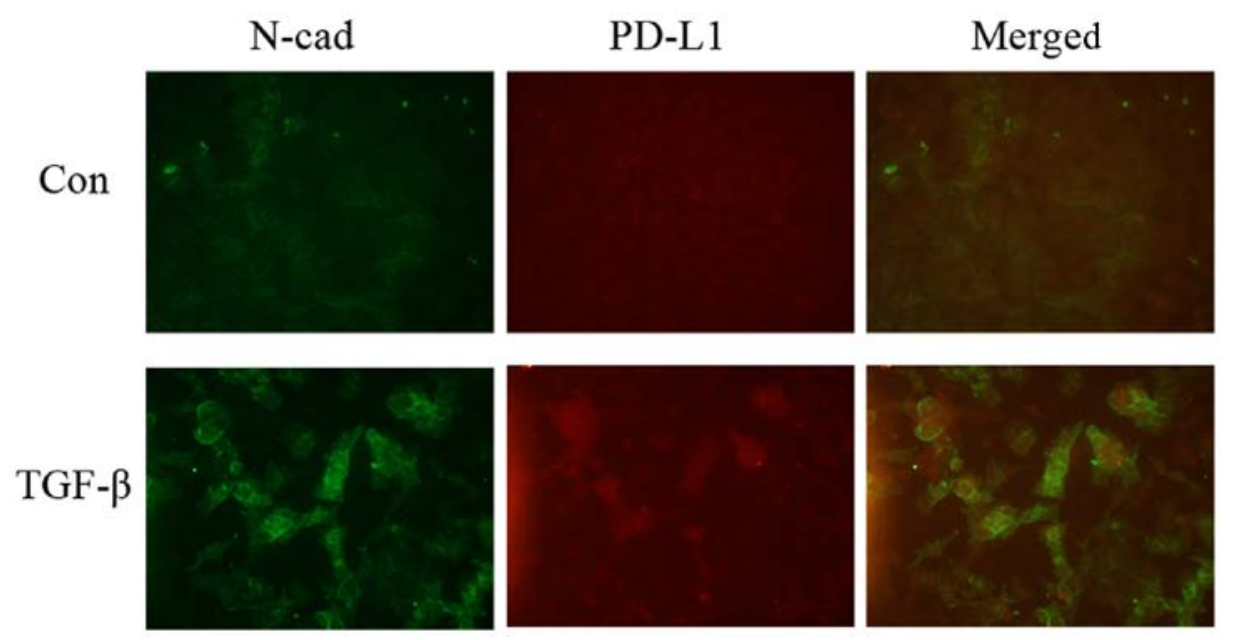

Figure 3. Immunofluorescence staining of TGF- $\beta$ treated A549 cells. Representative images of fluorescent immunohistochemical staining of N-cadherin (GFP) and PD-L1 (red) in control and TGF- $\beta$ treated A549 cells.

A

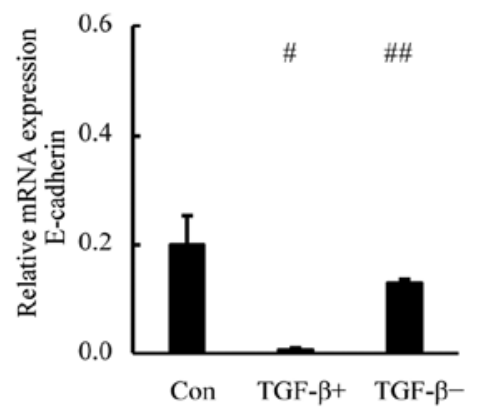

$\mathrm{D}$

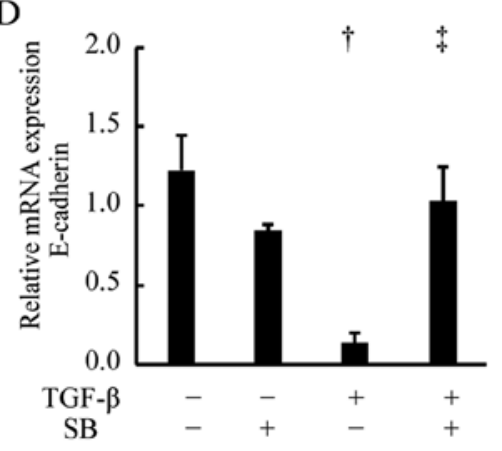

B

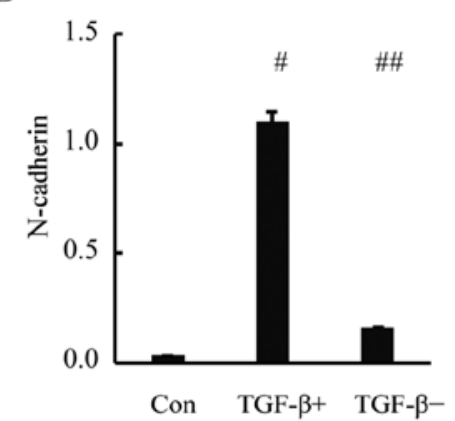

$\mathrm{E}$

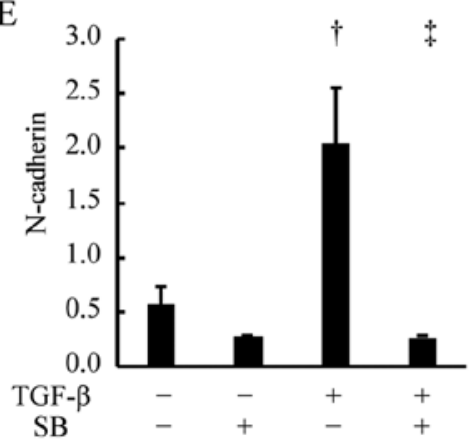

$\mathrm{C}$

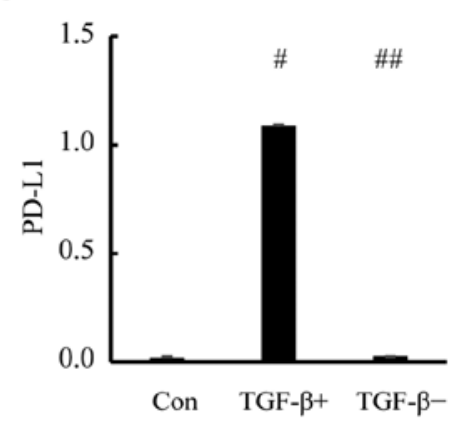

F

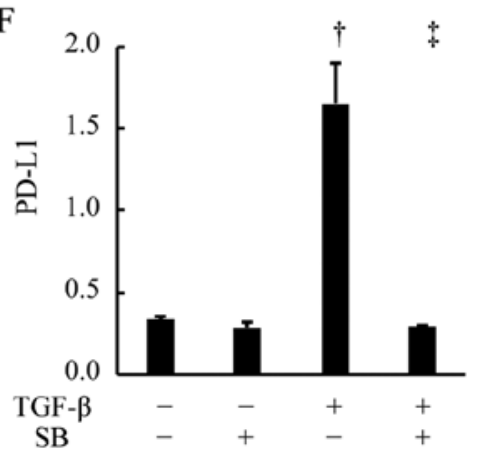

Figure 4. The PD-L1 expression is regulated by TGF- $\beta$ signaling. (A-C) The gene expression analysis of reversion assay. mRNA expression of A549 cells after TGF- $\beta 1$ treatment (TGF- $\left.\beta^{+}, 1 \mathrm{ng} / \mathrm{ml}\right)$ and A549 cells following removal of TGF- $\beta 1$ from the medium (TGF- $\left.\beta^{-}\right)$. The mRNA levels were normalized to the levels of GAPDH mRNA. $\left({ }^{\#} \mathrm{P}<0.01\right.$ vs. Con, ${ }^{\# \#} \mathrm{P}<0.01$ vs. TGF- $\left.\beta^{+}\right)$. (D-F) A549 cells were not treated (Con), treated with TGF- $\beta 1$ (TGF- $\beta^{+}, 2$ ng/ml), or treated with SB43152 (SB $\left.{ }^{+}, 10 \mu \mathrm{M}\right)$ plus TGF- $\beta 1(2 \mathrm{ng} / \mathrm{ml})$ for 3 days. Then, total RNA was extracted and real-time RT-PCR was performed to detect E-cadherin (E-cad), $\mathrm{N}$-cadherin (N-cad) and PD-L1. ( $\mathrm{P}<0.01$ vs. TGF- $\beta-\mathrm{SB}^{-},{ }^{\dagger} \mathrm{P}<0.01$ vs. TGF- $\left.\beta^{+} / \mathrm{SB}^{-}\right)$.

cells, whereas that of N-cadherin (as the mesenchymal marker) and PD-L1 were upregulated in a dose-dependent manner (Fig. 1A-C; $\mathrm{P}<0.05$ and $\mathrm{P}<0.01$ vs. Con). We also analyzed the PD-L1 gene expression in the human Caucasian bronchioalveolar carcinoma cell line $\mathrm{H} 358$ and obtained similar results (Fig. 1D-F; $\mathrm{P}<0.01$ vs. Con). Western blot analysis of A549 with TGF- $\beta 1$ revealed that equivalent differences were observed at the protein level (Fig. 2). Immunofluorescence analysis showed that the expression levels of PD-L1 and $\mathrm{N}$-cadherin were enhanced by TGF- $\beta$ treatment and those on the cell surface became co-localized (Fig. 3).
To determine whether there is a causal relationship between the PD-L1 expression and TGF- $\beta$ induced EMT, reversion assay was performed. We used RT-PCR assays to analyze the gene expression of PD-L1, E-cadherin and $\mathrm{N}$-cadherin in the cells under EMT and MET (Fig. 4A-C). The gene expression levels of PD-L1, E-cadherin and $\mathrm{N}$-cadherin changed with significant difference as compared with the pre-treatment levels (Con; control) $(\mathrm{P}<0.01$ vs. Con) and reverted to pre-treatment (TGF- $\left.\beta^{-}\right)$levels after the change to culture medium without TGF- $\beta$ ( ${ }^{\# \#} \mathrm{P}<0.01$ vs. TGF $\left.-\beta^{+}\right)$. These results suggest that the PD-L1 expression 


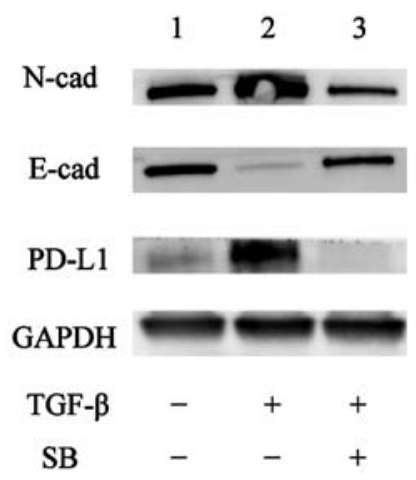

Figure 5. Western blot analysis of PD-L1 and EMT marker protein after TGF- $\beta$ treatment and TGF- $\beta 1$ receptor kinase inhibitor. A549 cells were not treated or treated with TGF- $\beta 1$ or TGF- $\beta 1$ plus SB 42152 for 3 days and then analyzed through western blotting for E-cadherin, N-cadherin, PD-L1 and GAPDH.

is regulated by the TGF- $\beta$ signaling and changes in parallel with the EMT status.

TGF- $\beta$ inhibitors block the expression of PD-L1. Next, to examine whether the PD-L1 expression was regulated through the TGF- $\beta$ signal pathway, we treated A549 cells for 2 days with medium alone (Fig. 4D-F, Con; TGF- $\beta^{-} / \mathrm{SB}^{-}$), the TGF- $\beta 1$ receptor kinase inhibitor; SB 431542 (Fig. 4D-F, TGF- $\beta$ - $/ \mathrm{SB}^{+}$), or TGF- $\beta 1$ plus SB 431542(Fig. 4D-F, TGF- $\beta^{+} / \mathrm{SB}^{+}$). Next, we examined PD-L1, E-cadherin and $\mathrm{N}$-cadherin expression by RT-PCR and western blot analysis. The RT-PCR results showed that the upregulation of PD-L1 and N-cadherin mRNA expression after TGF- $\beta 1$ treatment was suppressed by SB 431542 (Fig. $4 \mathrm{D}-\mathrm{F} ; \mathrm{P}<0.01$ vs. TGF- $\beta{ }^{-} / \mathrm{SB}^{-}, \mathrm{P}<0.01$ vs. TGF- $\left.\beta^{+} / \mathrm{SB}^{-}\right)$and the western blot analysis demonstrated that the PD-L1 protein level was suppressed by SB 431542 (Fig. 5, lane 2 vs. 3). Taken together, these results suggest that the PD-L1 expression is regulated through the TGF- $\beta$ signal pathway.

PD-L1 expression was enhanced by chemo-induced TGF- $\beta$ pathway. We previously reported that chemo-treatment increased the expression of TGF- $\beta$ and the autocrine TGF- $\beta$ induced EMT in A549 cells $(12,13)$. In this study, we hypothesized that chemo-treatment enhanced PD-L1 expression through the TGF- $\beta$ pathway. We evaluated the TGF- $\beta$, EMT markers and PD-L1 mRNA and protein expression in the A549 cells treated with carboplatin $(25 \mu \mathrm{M})$ for 4 days. The RT-PCR results indicated that, for E-cadherin, N-cadherin and PD-L1, the expression patterns of the control versus treatment subline (Fig. 6A-C) were similar to those for control vs. TGF- $\beta$ treatment $(\mathrm{P}<0.05$; vs. Con). Next, to examine whether PD-L1 upregulation under chemo-treatment was via TGF- $\beta$ pathway, we examined PD-L1 expression of the cells after treatment with carboplatin alone (CB) and carboplatin plus SB 43152 (CB+SB), by RT-PCR and western blot analysis. Fig. 6E showed the results of RT-PCR that PD-L1 gene expression after chemo-treatment (CB) increased significantly as compared with no-treated cell lines (Con). In contrast, the upregulation of the mRNA levels of PD-L1 and N-cadherin was attenuated by $\mathrm{SB} 431542$ (CB+SB) (Fig. 6D-E, $\mathrm{P}<0.05$ vs. Con, $\mathrm{P}<0.01$
Table II. Univariate and multivariate analyses of disease-free survival.

A, Univariate analysis of disease-free survival

\begin{tabular}{lccc}
\hline Factors & Hazard ratio & $95 \%$ CI & P-value \\
\hline Sex & & & \\
Male & 1 & $0.03-2.93$ & 0.58 \\
Female & 0.59 & & \\
Histology & & & \\
Adeno & 1 & $0.28-2.32$ & 0.66 \\
Sq & 0.79 & $0.02-2.31$ & 0.36 \\
other & 0.42 & & \\
p-Stage & 1 & & 0.1 \\
I+II & & & \\
III+IV & 2.29 & & \\
Down-stage & & & 0.16 \\
Positive & 1 & & \\
Negative & 2.27 & $0.73-10.03$ & \\
PD-L1 & & & \\
Low & 1 & & \\
High & 2.97 & & \\
\hline
\end{tabular}

B, Multivariate analysis of disease-free survival

\begin{tabular}{lccc}
\hline Factors & Hazard ratio & $95 \%$ CI & P-value \\
\hline PD-L1 & 1 & & \\
Low & 2.8 & $1.00-8.41$ & 0.04 \\
High & & & \\
Histology & 1 & & \\
Adeno & 0.8 & $0.26-2.42$ & 0.7 \\
Sq & 0.7 & $0.1-3.17$ & 0.69 \\
other & 1 & & \\
p-Stage & & & \\
I+II & & & \\
III+IV & 1 & $0.83-11.7$ & 0.09 \\
Down-stage & 1.95 & & 0.9 \\
Positve & & & \\
Negative & 1 & & \\
\hline
\end{tabular}

Adeno, adenocarcinoma; Sq, squamous cell carcinoma; CI, confidence interval.

vs. CB). While, TGF- $\beta$ mRNA expression was upregulated by carboplatin as previously described (12) (Fig. 6F; $\mathrm{P}<0.05$ vs. Con); however, TGF- $\beta$ upregulation with carboplatin treatment was not attenuated by SB 431542 . Fig. $6 \mathrm{~F}$ shows that there was no significant difference in TGF- $\beta$ mRNA levels between carboplatin alone (CB) and carboplatin plus SB 431542 treatment $(\mathrm{CB}+\mathrm{SB})$. Moreover, in western blot analysis, the expression of PD-L1 was upregulated by chemo-treatment in 

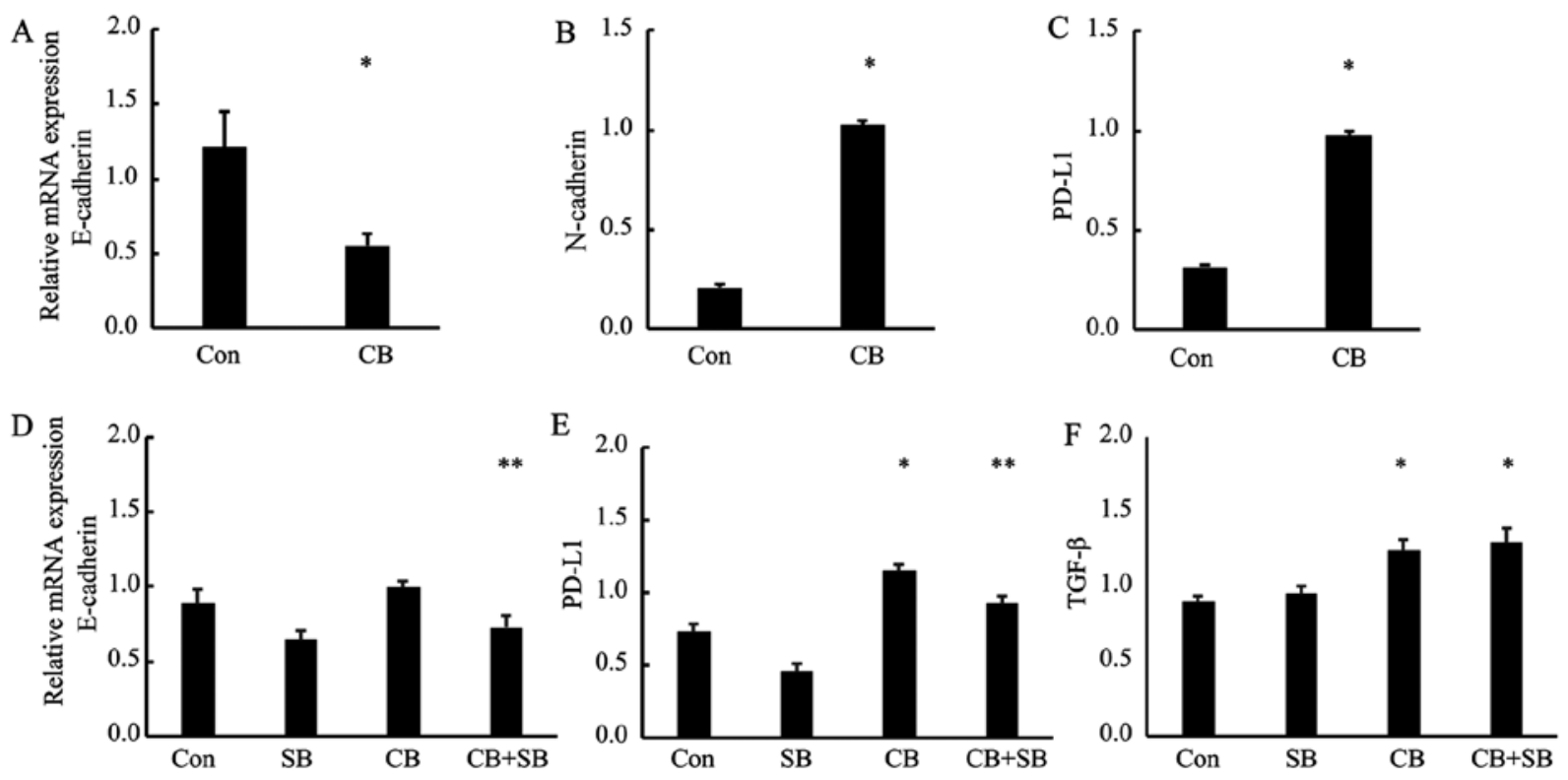

Figure 6. Chemo-treatment enhances PD-L1 expression via TGF- $\beta$ signaling. RT-PCR analysis of untreated A549 cells (Con) and those cells treated with carboplatin $(25 \mu \mathrm{M})$ for 4 days (CB). Total RNA was extracted from the untreated A549 cells (Con) and the cells treated with carboplatin (CB), and E-cadherin, $\mathrm{N}$-cadherin, PD-L1 and GAPDH transcripts were quantified by RT-PCR analysis (A-C). ("P<0.05 vs. Con). (D-F) Control A549 cells (Con), the cells treated with carboplatin alone (CB), and the cells treated with carboplatin and SB 43152 (SB; $10 \mu \mathrm{M})(\mathrm{CB}+\mathrm{SB})$ underwent RT-PCR analysis for quantification of TGF- $\beta$, N-cadherin, PD-L1 and GAPDH mRNA levels. (N-cad; ${ }^{*} \mathrm{P}<0.05$ vs. $\mathrm{CB}, \mathrm{PD}-\mathrm{L} 1$; ${ }^{*} \mathrm{P}<0.05$ vs. Con, ${ }^{* *} \mathrm{P}<0.01$ vs. CB, TGF- $\beta$; ${ }^{*} \mathrm{P}<0.05$ vs. Con).
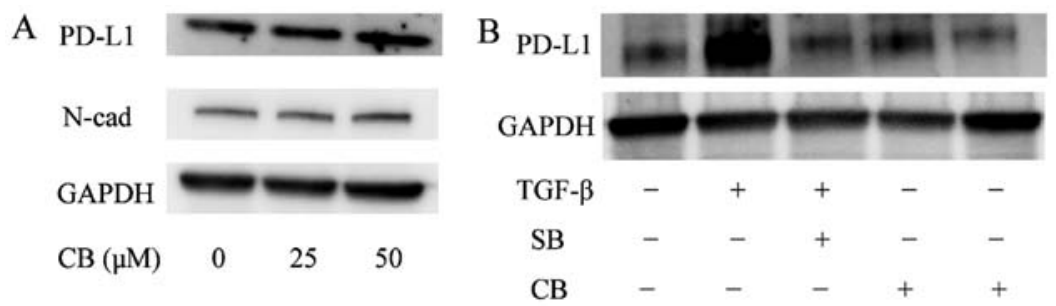

Figure 7. Chemo-treatment enhances PD-L1 protein expression via TGF- $\beta$ signaling. (A) Western blot analysis of the indicated proteins following carboplatin treatment (CB). Twenty-five and 50 indicate treatment with carboplatin at 25 and $50 \mu \mathrm{M}$, respectively. (B) Control A549 cells, the cells treated with carboplatin alone $\left(\mathrm{CB}^{+}\right)$, and cells treated with carboplatin and SB $43152(10 \mu \mathrm{M})\left(\mathrm{SB}^{+}\right)$underwent western blot analysis for quantification of PD-L1 and GAPDH levels.

a dose-dependent manner (Fig. 7A), and this upregulation was abolished by SB 431542 (Fig. 7B; lane 4 vs. 5). These results suggest that the PD-L1 gene expression is upregulated through the chemo-induced TGF- $\beta$ pathway.

High expression of PD-L1 in non-small cell lung cancer cases after induction chemotherapy. To elucidate the relationship between PD-L1 and EMT status in clinical samples of NSCLC, we performed IHC staining of clinical specimens obtained from samples resected from 28 patients with NSCLC after induction chemotherapy.

The patient characteristics are listed in Table I. IHC staining was used to analyze the expression of EMT markers, TGF- $\beta$ and PD-L1. Fig. 8A shows the representative images of low expression of PD-L1 and Fig. 8B shows those of high expression of PD-L1. Fig. 8C-E shows the representative images of E-cadherin, $\mathrm{N}$-cadherin and TGF- $\beta$ positive IHC. A total of 28 patients with NSCLC underwent induction chemotherapy followed by complete surgical resection. All cases underwent carboplatin or cisplatin-based doublet chemotherapy. The patients comprised 22 men and 6 women with a mean age of 63.1 .
Eight cases (28.6\%) showed a positive EMT change. Of them, $7(87.5 \%)$ showed positive results for PD-L1 staining. The proportion of EMT-change positive cases among PD-L1high expression cases was significantly higher than that among PD-L1-low expression cases (Fig. 9A, Pearson's chi-square test; $\mathrm{P}=0.01)$. In TGF- $\beta$ IHC analysis, there were also significant relationships between PD-L1 expression and TGF- $\beta$ expression (Fig. 9B, Pearson's chi-square test; $\mathrm{P}=0.04$ ). The DFS (measured as no recurrence of cancer) in PD-L1-high expression cases was significantly worse than that of PD-L1-low expression cases (Fig. 9C, $\mathrm{P}=0.02$; log-rank test); however, the OS rate was not significantly related to PD-L1 status (Fig. 9D, $\mathrm{P}=0.2$; log-rank test).

Univariate and multivariate analyses showed that high PD-L1 expression was an independent prognostic factor for NSCLC surgically resected after induction chemotherapy (Table II).

\section{Discussion}

In the present study, we showed that PD-L1 was regulated by TGF- $\beta$, and that chemo-treatment enhanced the PD-L1 expres- 

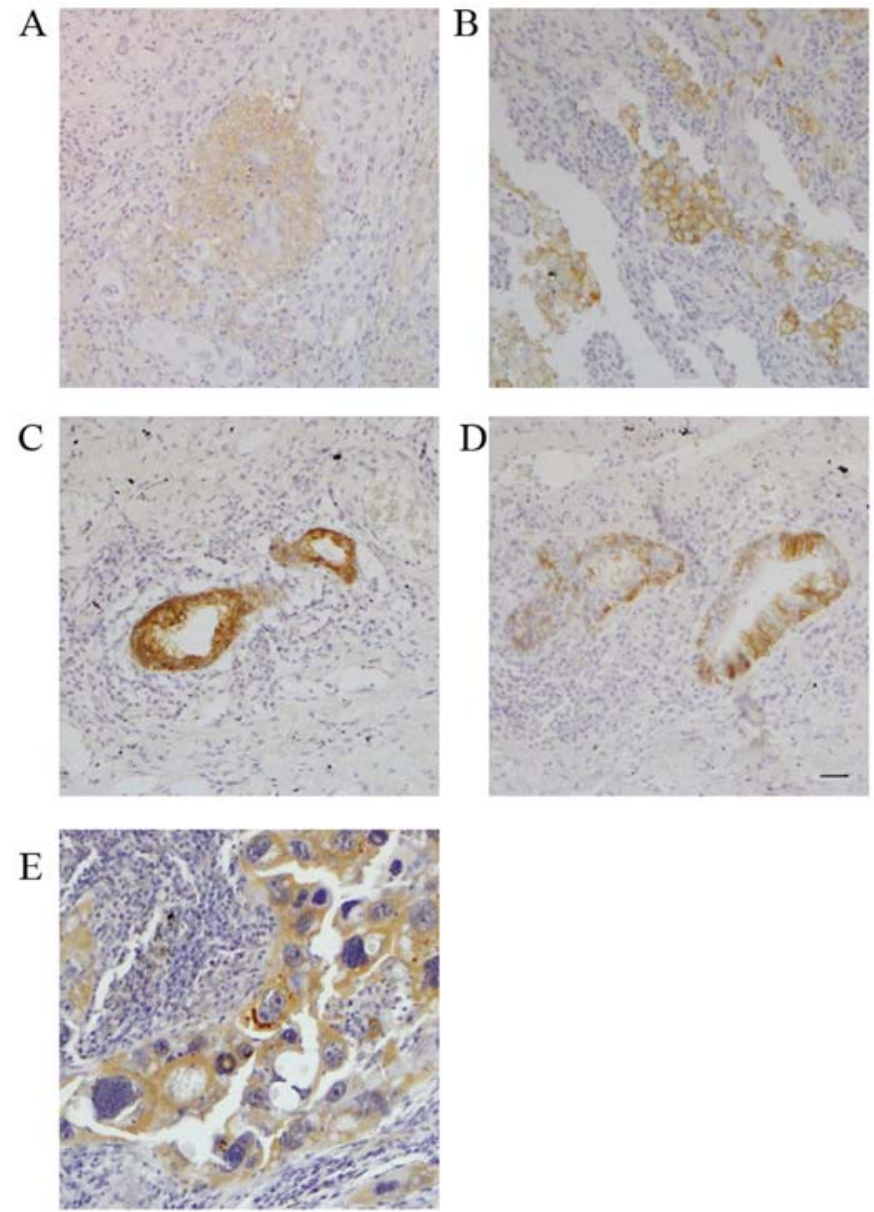

Figure 8. Immunohistochemical staining analysis of PD-L1 and EMT markers. Representative images of immunohistochemical staining of PD-L1 and EMT marker. (A) Weak staining intensity of PD-L1. (B) Strong staining intensity of PD-L1. The representative images of positive E-cadherin $\mathrm{N}$-cadherin and TGF- $\beta$ IHC staining $(\mathrm{C}$; E-cadherin, D; N-cadherin, E; TGF- $\beta$ ). Scale bar, $100 \mu \mathrm{m}$.

sion through the chemo-induced TGF- $\beta$ signal pathway in NSCLC cell lines. We also performed IHC staining of surgically resected NSCLC after induction chemotherapy and thus revealed a significant relationship between PD-L1 expression and EMT status.

To overcome advanced NSCLC, surgery and chemotherapy are the most important therapeutic options; however, their results are far from satisfactory. Immunotherapy has been recently developed as another option; in particular, anti-PD-1/PD-L1 blockade agents have exhibited dramatic antitumor efficacy in clinical trials for patients with a variety of cancer types (14). However, immunotherapy likewise is associated with several problems to be solved, as outlined below.

The first problem is the selection of suitable patients. The current selection method depends on PD-L1 IHC results as a predictor of therapeutic effect. PD-L1 expression by IHC has been also reported to be a useful prognostic indicator (15); however, staining intensity and sensitivity vary according to the type of PD-L1 IHC antibody $(15,16)$. The next problem is when to start this immunotherapy. Understanding the mechanism of PD-L1 expression will yield important information regarding potential solutions to these problems. To elucidate this mechanism, we focused on EMT.
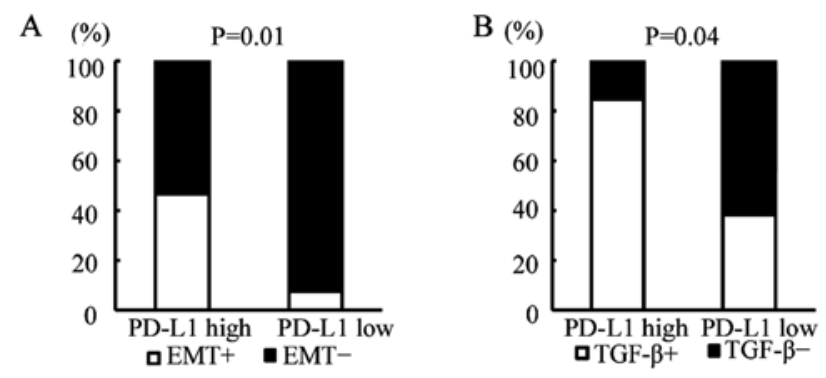

$\mathrm{C}$

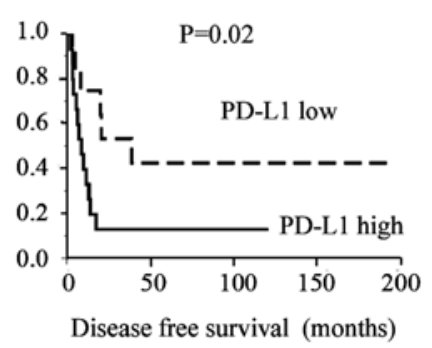

$\mathrm{D}$

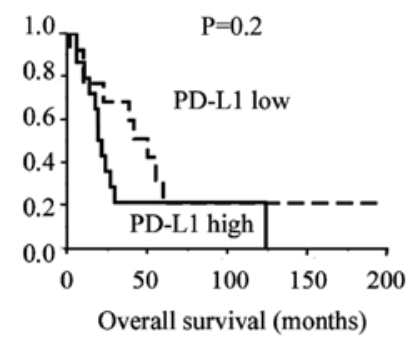

Figure 9. The relationships between PD-L1 expression and EMT markers, and Kaplan-Meier survival curve according to PD-L1 expression. (A) Proportions of cells staining for EMT status in PD-L1 expression intensity (Pearson's chisquare test). (B) Proportion of cells staining for TGF- $\beta$ according to PD-L1 expression intensity (Pearson's chi-square test). (C) Disease-free survival rates and (D) overall survival rates according to tumor expression of PD-L1.

EMT plays a crucial role in cancer progression, and this phenomenon can be found in this cancer microenvironment. EMT is promoted by TGF- $\beta$ secreted from not only the cancer cell itself but also from other cell types, and it gives cancer cells the invasive and metastatic abilities necessary for successful metastasis $(7,8,12,17)$. In the present study, we demonstrated that PD-L1 expression in cancer cells was regulated by TGF- $\beta$ signal pathway. Other groups showed a significant relationship between PD-L1 expression and EMT status by IHC staining analysis on several malignancies including NSCLC $(18,19)$. Together, these data support our notion that the TGF- $\beta$ pathway is an important regulator of PD-L1 expression, and EMT markers may be useful for selecting patients that would likely benefit from immunotherapy.

We previously reported that chemo-treatments increased TGF- $\beta$ production and autocrine TGF- $\beta$ induced EMT in human lung adenocarcinoma cell lines $(7,12)$. Based on our previous results, we showed that chemo-treatment enhanced PD-L1 expression through the chemo-induced TGF- $\beta$ signal pathway. Moreover, from the IHC analysis, we revealed the significant relationship between PD-L1 expression and EMT status of resected samples after induction chemotherapy. Zhang et al (20) demonstrated that chemo-preventive agents induced PD-L1 in human breast cancer cells and promoted PD-L1-mediated INF- $\gamma$ by T-cell apoptosis. Hecht et al (21) also showed that PD-L1 expressing cells in rectal adenocarcinoma were upregulated after chemoradiotherapy (CRT) as compared with before CRT. Other groups have shown that several stimuli, such as hypoxia and radiation induction, enhance PD-L1 expression (22-24). Those results suggested that PD-L1 is enhanced by chemo-treatment and the PD-L1 blockade after chemotherapy or in combination with chemo- 
therapy could be a more effective anticancer activity than the monotherapy.

This study has some limitations. The limitation is the small sample size analyzed. In our hospital, adjuvant chemoradiation therapy was usually performed in most of advanced cases. As mentioned above, the radiation, like chemotherapy, enhances the expression of PD-L1 (22-24). Since the effect of radiation cannot be ignored, we analyzed only cases after induction chemotherapy alone. Moreover, the predictive role of PD-L1 expression is still controversial. Our results showed the PD-L1 expression was a prognostic indicator. However, our sample size was small as mentioned. Therefore, further investigation will be needed.

In conclusion, the present study provides new and important information regarding the mechanism of PD-L1 expression. The results suggest that EMT markers could be a surrogate marker for the selection of patients for immunotherapy and that a combination of immunotherapy and chemotherapy could be a new therapeutic option to overcome NSCLC.

\section{Acknowledgements}

The present study was supported by KAKENHI (Grantsin-Aid for Scientific Research) 16K10680.

\section{References}

1. Bordon Y: Immunotherapy: Checkpoint parley. Nat Rev Cancer 15: 3, 2015.

2. Reck M, Rodríguez-Abreu D, Robinson AG, Hui R, Csőszi T, Fülöp A, Gottfried M, Peled N, Tafreshi A, Cuffe S, et al; KEYNOTE-024 Investigators: Pembrolizumab versus chemotherapy for PD-L1-positive non-small-cell lung cancer. N Engl J Med 375: 1823-1833, 2016.

3. Brahmer J, Reckamp KL, Baas P, Crinò L, Eberhardt WE, Poddubskaya E, Antonia S, Pluzanski A, Vokes EE, Holgado E, et al: Nivolumab versus docetaxel in advanced squamous-cell non-small-cell lung cancer. N Engl J Med 373: 123-135, 2015.

4. Weber JS, D'Angelo SP, Minor D, Hodi FS, Gutzmer R, Neyns B, Hoeller C, Khushalani NI, Miller WH Jr,Lao CD, et al: Nivolumab versus chemotherapy in patients with advanced melanoma who progressed after anti-CTLA-4 treatment (CheckMate 037): A randomised, controlled, open-label, phase 3 trial. Lancet Oncol 16: 375-384, 2015

5. Iwai Y, Ishida M, Tanaka Y, Okazaki T, Honjo T and Minato N: Involvement of PD-L1 on tumor cells in the escape from host immune system and tumor immunotherapy by PD-L1 blockade. Proc Natl Acad Sci USA 99: 12293-12297, 2002.

6. Yang Y, Pang Z, Ding N, Dong W, Ma W, Li Y, Du J and Liu Q The efficacy and potential predictive factors of PD-1/PD-L1 blockades in epithelial carcinoma patients: A systematic review and meta analysis. Oncotarget 7: 74350-74361, 2016.

7. Shintani Y, Okimura A, Sato K, Nakagiri T, Kadota Y, Inoue M, Sawabata N, Minami M, Ikeda N, Kawahara K, et al: Epithelial to mesenchymal transition is a determinant of sensitivity to chemoradiotherapy in non-small cell lung cancer. Ann Thorac Surg 92: 1794-1804, discussion 1804, 2011.

8. Shintani Y, Abulaiti A, Kimura T, Funaki S, Nakagiri T, Inoue M, Sawabata N, Minami M, Morii E and Okumura M: Pulmonary fibroblasts induce epithelial mesenchymal transition and some characteristics of stem cells in non-small cell lung cancer. Ann Thorac Surg 96: 425-433, 2013.
9. Koh J, Go H, Keam B, Kim MY, Nam SJ, Kim TM, Lee SH, Min HS, Kim YT, Kim DW, et al: Clinicopathologic analysis of programmed cell death-1 and programmed cell death-ligand 1 and 2 expressions in pulmonary adenocarcinoma: Comparison with histology and driver oncogenic alteration status. Mod Pathol 28: 1154-1166, 2015.

10. Shintani Y, Maeda M, Chaika N, Johnson KR and Wheelock MJ: Collagen I promotes epithelial-to-mesenchymal transition in lung cancer cells via transforming growth factor-beta signaling. Am J Respir Cell Mol Biol 38: 95-104, 2008.

11. McCarty KS Jr, Szabo E, Flowers JL, Cox EB, Leight GS, Miller L, Konrath J, Soper JT, Budwit DA, Creasman WT, et al: Use of a monoclonal anti-estrogen receptor antibody in the immunohistochemical evaluation of human tumors. Cancer Res 46 (Suppl): 4244s-4248s, 1986.

12. Shintani Y, Fujiwara A, Kimura T, Kawamura T, Funaki S, Minami $M$ and Okumura M: IL-6 decreted from cancerassociated fibroblasts mediates chemoresistance in NSCLC by increasing epithelial-mesenchymal transition signaling. J Thorac Oncol 11: 1482-1492, 2016.

13. Abulaiti A, Shintani Y, Funaki S, Nakagiri T, Inoue M, Sawabata N, Minami M and Okumura M: Interaction between non-small-cell lung cancer cells and fibroblasts via enhancement of TGF- $\beta$ signaling by IL-6. Lung Cancer 82: 204-213, 2013.

14. Borghaei H, Paz-Ares L, Horn L, Spigel DR, Steins M, Ready NE, Chow LQ, Vokes EE, Felip E, Holgado E, et al: Nivolumab versus docetaxel in advanced nonsquamous non-small-cell lung cancer. N Engl J Med 373: 1627-1639, 2015.

15. Schats KA, Van VréEA, De Schepper S, Boeckx C, Schrijvers DM, Waelput W, Fransen E, Vanden Bempt I, Neyns B, De Meester I, et al: Validated programmed cell death ligand 1 immunohistochemistry assays (E1L3N and SP142) reveal similar immune cell staining patterns in melanoma when using the same sensitive detection system. Histopathology 70: 253-263, 2017.

16. Smith J, Robida MD, Acosta K, Vennapusa B, Mistry A, Martin G, Yates A and Hnatyszyn HJ: Quantitative and qualitative characterization of Two PD-L1 clones: SP263 and E1L3N. Diagn Pathol 18: 44, 2016.

17. Thomson S, Petti F, Sujka-Kwok I, Mercado P, Bean J, Monaghan M, Seymour SL, Argast GM, Epstein DM and Haley JD: A systems view of epithelial-mesenchymal transition signaling states. Clin Exp Metastasis 28: 137-155, 2011.

18. Shimoji M, Shimizu S, Sato K, Suda K, Kobayashi Y, Tomizawa $\mathrm{K}$, Takemoto $\mathrm{T}$ and Mitsudomi T: Clinical and pathologic features of lung cancer expressing programmed cell death ligand 1 (PD-L1). Lung Cancer 98: 69-75, 2016.

19. Ock CY, Kim S, Keam B, Kim M, Kim TM, Kim JH, Jeon YK, Lee JS, Kwon SK, Hah JH, et al: PD-L1 expression is associated with epithelial-mesenchymal transition in head and neck squamous cell carcinoma. Oncotarget 7: 15901-15914, 2016

20. Zhang P, Su DM, Liang M and Fu J: Chemopreventive agents induce programmed death-1-ligand 1 (PD-L1) surface expression in breast cancer cells and promote PD-L1-mediated T cell apoptosis. Mol Immunol 45: 1470-1476, 2008.

21. Hecht M, Büttner-Herold M, Erlenbach-Wünsch K, Haderlein M, Croner R, Grützmann R, Hartmann A, Fietkau R and Distel LV: PD-L1 is upregulated by radiochemotherapy in rectal adenocarcinoma patients and associated with a favourable prognosis. Eur J Cancer 65: 52-60, 2016.

22. Noman MZ, Desantis G, Janji B, Hasmim M, Karray S, Dessen P, Bronte V and Chouaib S: PD-L1 is a novel direct target of HIF-1 $\alpha$, and its blockade under hypoxia enhanced MDSC-mediated T cell activation. J Exp Med 211: 781-790, 2014.

23. Dovedi SJ, Adlard AL, Lipowska-Bhalla G, McKenna C, Jones S, Cheadle EJ, Stratford IJ, Poon E, Morrow M, Stewart R, et al: Acquired resistance to fractionated radiotherapy can be overcome by concurrent PD-L1 blockade. Cancer Res 74: 5458-5468, 2014.

24. Deng L, Liang $H$, Burnette $B$, Beckett $M$, Darga $T$, Weichselbaum RR and Fu YX: Irradiation and anti-PD-L1 treatment synergistically promote antitumor immunity in mice. J Clin Invest 124: 687-695, 2014. 